34. B. Peucker-Ehrenbrink, G. Ravizza, Terra Nova 12, 205 (2000).

35. B. Peucker-Ehrenbrink, G. Ravizza, in The Geologic Time Scale, F. M. Gradstein, ]. G. Ogg, M. D. Schmitz, Eds. (Cambridge Univ. Press, Cambridge, 2012) chapter $8 ; 10.1016 /$ B978-0-444-59425-9.00008-1.

36. ]. M. Edmond, Science 258, 1594 (1992).

37. B. Peucker-Ehrenbrink, R. E. Hannigan, Geology 28, 475 (2000).

38. E. M. Griffith, A. Paytan, K. Caldeira, T. D. Bullen, E. Thomas, Science 322, 1671 (2008).

39. C. H. Lear, H. Elderfield, P. A. Wilson, Earth Planet. Sci. Lett. 208, 69 (2003).

40. A. Paytan, M. Kastner, D. Campbell, M. H. Thiemens, Science 304, 1663 (2004).

41. R. A. Berner, A. C. Lasaga, R. M. Garrels, Am. J. Sci. 283 641 (1983).

42. F. T. Mackenzie, L. R. Kump, Science 270, 586 (1995).

43. V. Courtillot, P. R. Renne, C. R. Geosci. 335, 1 (2003).

44. D. V. Kent, G. Muttoni, Proc. Natl. Acad. Sci. U.S.A. 105, 16065 (2008).
45. S. R. Gislason, E. H. Oelkers, A. Snorrason, Geology 34, 49 (2006).

46. C. Jaramillo, M. J. Rueda, G. Mora, Science 311, 1893 (2006).

47. M. T. Clementz, J. O. Sewall, Science 332, 455 (2011).

48. L. W. Alvarez, W. Alvarez, F. Asaro, H. V. Michel, Science 208, 1095 (1980).

49. P. Schulte et al., Science 327, 1214 (2010).

50. J. W. Farrell, S. C. Clemens, L. Peter Gromet, Geology 23, 403 (1995).

51. E. E. Martin, H. D. Scher, Earth Planet. Sci. Lett. 220, 25 (2004).

52. E. E. Martin, N. J. Shackleton, ]. C. Zachos, B. P. Flower, Paleoceanography 14, 74 (1999).

53. D. A. Hodell et al., Geochem. Geophys. Geosyst. 8, Q09001 (2007)

54. T. Westerhold et al., Palaeogeogr. Palaeoclimatol. Palaeoecol. 257, 377 (2008).

Acknowledgments: We thank the U.S. National Science Foundation (MG\&G), American Chemical Society (Petroleum Research Fund), and The Francis Eppes Society of Florida State
University for providing financial support. We sincerely thank S. Clemens, D. Hodell, E. Hathorne, E. Martin, E. Thomas,

W. Landing, and H. Spero for providing samples. The manuscript greatly benefited from the constructive reviews of $M$. Bender, V. Salters, M. Humayun, A. Paytan, P. Pogge von Strandmann, and an anonymous reviewer. We are very thankful to B. Peucker-Ehrenrbrink and G. Ravizza for providing the osmium isotope data from GTS 2012 (in press). We also thank H. Elderfield, I. N. McCave, and A. Galy for helpful discussions. Tabulated data are provided on Science Online.

\section{Supporting Online Material}

www.sciencemag.org/cgi/content/full/science.1214697/DC1

Materials and Methods

SOM Text

Figs. S1 to S11

Table S1

References (55-157)

30 September 2011; accepted 17 January 2012

Published online 26 January 2012;

$10.1126 /$ science. 1214697

\title{
A Systematic Survey of Loss-of-Function Variants in Human Protein-Coding Genes
}

Daniel G. MacArthur, ${ }^{1,2 *}$ Suganthi Balasubramanian, ${ }^{3,4}$ Adam Frankish, ${ }^{1}$ Ni Huang, ${ }^{1}$ James Morris, ${ }^{1}$ Klaudia Walter, ${ }^{1}$ Luke Jostins, $^{1}$ Lukas Habegger, ${ }^{3,4}{ }^{3}{ }^{\circ}$ oseph K. Pickrell, ${ }^{5}$ Stephen B. Montgomery, ${ }^{6,7}$ Cornelis A. Albers, ${ }^{1,8}$ Zhengdong D. Zhang, ${ }^{9}$ Donald F. Conrad, ${ }^{10}$ Gerton Lunter, ${ }^{11}$ Hancheng Zheng, ${ }^{12}$ Qasim Ayub, ${ }^{1}$ Mark A. DePristo, ${ }^{13}$ Eric Banks, ${ }^{13}$ Min Hu, ${ }^{1}$ Robert E. Handsaker, ${ }^{13,14}$ jeffrey A. Rosenfeld, ${ }^{15}$ Menachem Fromer, ${ }^{13}$ Mike Jin, ${ }^{3}$ Xinmeng Jasmine $\mathrm{Mu}^{3,4}$ Ekta Khurana, ${ }^{3,4} \mathrm{Kai} \mathrm{Ye}^{16}{ }^{16}$ Mike Kay, ${ }^{1}$ Gary lan Saunders, ${ }^{1}$ Marie-Marthe Suner, ${ }^{1}$ Toby Hunt, ${ }^{1}$ If H. A. Barnes, ${ }^{1}$ Clara Amid, ${ }^{1,17}$ Denise R. Carvalho-Silva, ${ }^{1}$ Alexandra H. Bignell, ${ }^{1}$ Catherine Snow, ${ }^{1}$ Bryndis Yngvadottir, ${ }^{1}$ Suzannah Bumpstead, ${ }^{1}$ David N. Cooper, ${ }^{18}$ Yali Xue, ${ }^{1}$ Irene Gallego Romero, ${ }^{1,5} 1000$ Genomes Project Consortium, Jun Wang, ${ }^{12}$ Yingrui Li, ${ }^{12}$ Richard A. Gibbs, ${ }^{19}$ Steven A. McCarroll, ${ }^{13,14}$

Emmanouil T. Dermitzakis, ${ }^{7}$ Jonathan K. Pritchard, ${ }^{5,20}$ Jeffrey C. Barrett, ${ }^{1}$ Jennifer Harrow, ${ }^{1}$

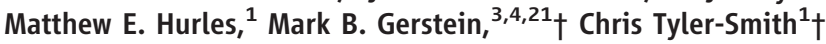

Genome-sequencing studies indicate that all humans carry many genetic variants predicted to cause loss of function (LoF) of protein-coding genes, suggesting unexpected redundancy in the human genome. Here we apply stringent filters to 2951 putative LoF variants obtained from 185 human genomes to determine their true prevalence and properties. We estimate that human genomes typically contain $\sim 100$ genuine LoF variants with $\sim 20$ genes completely inactivated. We identify rare and likely deleterious LoF alleles, including 26 known and 21 predicted severe disease-causing variants, as well as common LoF variants in nonessential genes. We describe functional and evolutionary differences between LoF-tolerant and recessive disease genes and a method for using these differences to prioritize candidate genes found in clinical sequencing studies.

$\mathrm{G}$ enetic variants predicted to severely disrupt protein-coding genes, collectively known as loss-of-function (LoF) variants, are of considerable scientific and clinical interest. Traditionally, such variants have been regarded as rare and having a high probability of being deleterious, on the basis of their well-established causal roles in severe Mendelian diseases such as cystic fibrosis and Duchenne muscular dystrophy. However, recent studies examining the complete genomes of apparently healthy subjects have suggested that such individuals carry at least 200 $(1,2)$ and perhaps as many as $800(3)$ predicted LoF variants. These numbers imply a previously unappreciated robustness of the human genome to gene-disrupting mutations and have important implications for the clinical interpretation of human genome-sequencing data.

Comparison of reported LoF variants between published genomes is complicated by differences in sequencing technology, variant-calling algorithms, and gene annotation sets between studies $(4,5)$, and by the expectation that LoF variants will be highly enriched for false positives. The basis for this predicted enrichment is that strong negative natural selection is expected to act against the majority of variants inactivating proteincoding genes, thereby reducing the amount of true variation at these sites relative to the genome average, whereas sequencing error is expected to be approximately uniformly distributed; as a result, highly functionally constrained sites should show lower levels of observed polymorphism and substantially higher false-positive rates (4). To date, no large-scale attempt has been made to validate the LoF variants reported in published human genome sequences.

LoF variants found in healthy individuals will fall into several overlapping categories: severe recessive disease alleles in the heterozygous state; alleles that are less deleterious but nonetheless have an impact on phenotype and disease risk; benign LoF variation in redundant genes; genuine variants that do not seriously disrupt gene function; and, finally, a wide variety of sequencing

${ }^{1}$ Wellcome Trust Sanger Institute, Hinxton CB10 1SA, UK. ${ }^{2}$ Discipline of Paediatrics and Child Health, University of Sydney, Sydney, NSW 2006, Australia. ${ }^{3}$ Program in Computational Biology and Bioinformatics, Yale University, New Haven, CT 06520 USA. ${ }^{4}$ Department of Molecular Biophysics and Biochemistry, Yale University, New Haven, CT 06520, USA. ${ }^{5}$ Department of Human Genetics, University of Chicago, Chicago, IL 60637, USA. ${ }^{6}$ Departments of Pathology and Genetics, Stanford University, Stanford, CA 94305-5324, USA. ${ }^{7}$ Department of Genetic Medicine and Development, University of Geneva Medical School, 1211 Geneva 4, Switzerland. ${ }^{8}$ Department of Haematology, University of Cambridge and NHS Blood and Transplant, Cambridge CB2 OPT, UK. 'Department of Genetics, Albert Einstein College of Medicine, Bronx, NY 10461, USA. ${ }^{10}$ Department of Genetics, Washington University School of Medicine, Saint Louis, MO 63110, USA. ${ }^{11}$ Wellcome Trust Centre for Human Genetics, University of Oxford, Oxford OX3 7BN, UK. ${ }^{12}$ BGI-Shenzhen, Shenzhen 518083, China. ${ }^{13}$ Program in Medical and Population Genetics, Broad Institute of Harvard and MIT, Cambridge, MA 02142, USA. ${ }^{14}$ Department of Genetics, Harvard Medical School, Boston, MA 02115, USA. ${ }^{15}$ IST/High Performance and Research Computing, University of Medicine and Dentistry of New Jersey, Newark, N] 07103, USA. ${ }^{16}$ Molecular Epidemiology Section, Leiden University Medical Center, 2300 RC Leiden, Netherlands. ${ }^{17}$ The European Nucleotide Archive, European Molecular Biology Laboratory-European Bioinformatics Institute, Hinxton CB10 1SD, UK. ${ }^{18}$ Institute of Medical Genetics, Schoo of Medicine, Cardiff University, Heath Park, Cardiff CF14 4XN, UK. ${ }^{19}$ Human Genome Sequencing Center, Baylor College of Medicine, Houston, TX 77030, USA. ${ }^{20}$ Howard Hughes Medical Institute, University of Chicago, Chicago, IL 60637, USA. ${ }^{21} \mathrm{De}$ partment of Computer Science, Yale University, New Haven, CT, USA.

*To whom correspondence should be addressed. E-mail: macarthur@atgu.mgh.harvard.edu

†These authors contributed equally to this work. 
and annotation artifacts. Distinguishing between these categories will be crucial for the complete functional interpretation of human genome sequences.

Obtaining and filtering candidate LoF variants. We identified 2951 candidate LoF variants using whole-genome sequencing data from 185 individuals analyzed as part of the pilot phase of the 1000 Genomes Project (2), as well as detailed analysis of high-coverage whole-genome sequencing data from a single anonymous European individual (6). The individuals represented three population groups: Yoruba individuals from Ibadan, Nigeria (YRI); 60 individuals of Northern and Western European origin from Utah (CEU); and 30 Chinese individuals from Beijing and 30 Japanese individuals from Tokyo who were analyzed jointly $(\mathrm{CHB}+\mathrm{JPT})$.
We adopted a definition for LoF variants expected to correlate with complete loss of function of the affected transcripts: stop codonintroducing (nonsense) or splice site-disrupting single-nucleotide variants (SNVs), insertion/deletion (indel) variants predicted to disrupt a transcript's reading frame, or larger deletions removing either the first exon or more than $50 \%$ of the proteincoding sequence of the affected transcript. We further subdivided these variants into "full" LoF variants predicted to affect all known proteincoding transcripts of the affected gene and "partial" variants affecting only a fraction of known coding transcripts. All annotation was performed against the Gencode v3b annotation (7) with the algorithm VAT (8).

We then subjected our candidate list to a series of stringent informatic and experimental val-
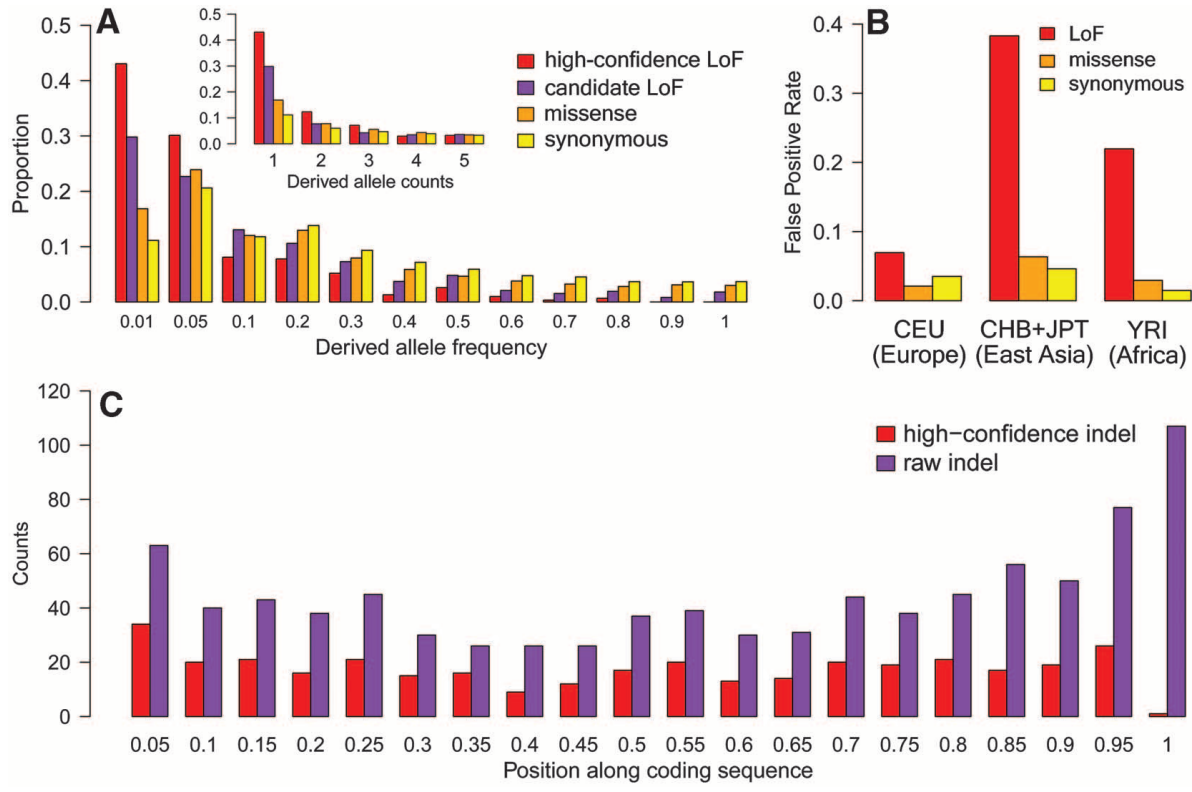

Fig. 1. (A) Derived allele frequency distribution in the CEU population for raw and high-confidence LoF variants, compared to missense and synonymous coding variants. (Inset) Distribution of the proportion of SNVs in each class at low allele counts (1 to 5). (B) False-positive rates (based on independent array genotyping) for LoF variants filtered for annotation artifacts and frequencymatched missense and synonymous SNVs. (C) Distribution of frameshift indels along the coding region of affected genes, before and after filtering. idation steps (9). Informatic filtering was based on local sequence context (such as the presence of highly repetitive sequence), gene annotation (such as variants affecting noncanonical splice sites or located close to the end of the affected open reading frame), analysis of the effects of nearby variants (such as neighboring SNVs altering the predicted functional effect of the candidate LoF variant), and measures of sequence read mapping and quality (fig. S1). Where possible, thresholds for filtering were derived from the experimental validation experiments below.

We validated all candidate LoF SNVs and indels that were not excluded by other filters and for which we could design assays $(n=1877)$ with experimental genotyping using three Illumina genotyping arrays and 819 custom Sequenom assays run, where possible, on all 185 samples from the low- and high-coverage 1000 Genomes pilot projects. Large deletions had previously been subjected to extensive validation (10). All LoF variants identified in NA12878 were assessed by comparison with independent 454 sequencing and array-based data from the same individual, as well as targeted capillary sequencing of variants in highly repetitive regions. Finally, 786 variants were reexamined by complete manual reannotation of the 689 affected gene models by experienced curators, using the HAVANA annotation pipeline (7), to identify annotation errors and flag variants unlikely to profoundly affect gene function. All 589 candidate LoF variants identified in NA12878 were subjected to independent genotype validation and complete gene model reannotation.

As expected, the proportion of likely sequencing and annotation errors in the initial candidate set was high, with overlapping sets of 25.0, 26.8, and $11.1 \%$ examined LoF variants being excluded as representing likely sequencing or mapping errors, annotation or reference sequence errors, and variants unlikely to cause genuine $\mathrm{LoF}$, respectively. Candidate LoF variants removed by filtering tended to be more common than highconfidence variants (Fig. 1A). False-positive rates due to sequencing errors (Fig. 1B) were higher for $\mathrm{LoF}$ variants than for missense and synonymous variants in the $\mathrm{CHB}+\mathrm{JPT}$ and YRI populations

Table 1. Numbers of LoF variants before and after filtering. Total numbers of candidate LoF variants and average number of LoF sites per individual (homozygous sites in parentheses) are shown for each LoF class. For large deletions, numbers represent total number of genes predicted to be inactivated.

Before filtering

After filtering

\begin{tabular}{|c|c|c|c|c|c|c|c|c|c|c|}
\hline \multirow[t]{2}{*}{$\begin{array}{l}\text { Variant } \\
\text { type }\end{array}$} & \multirow{2}{*}{ Total } & \multicolumn{3}{|c|}{$\begin{array}{l}\text { 1000G low-coverage } \\
\text { average per individual }\end{array}$} & \multirow{2}{*}{ NA12878 } & \multirow{2}{*}{ Total } & \multicolumn{3}{|c|}{$\begin{array}{l}\text { 1000G low-coverage } \\
\text { average per individual }\end{array}$} & \multirow[t]{2}{*}{ NA12878 } \\
\hline & & CEU & $\mathrm{CHB}+\mathrm{JPT}$ & YRI & & & CEU & $\mathrm{CHB}+\mathrm{JPT}$ & YRI & \\
\hline Stop & 1111 & 85.7 (21.8) & $113.4(26.7)$ & $109.1(23.7)$ & $115(25)$ & 565 & $26.2(5.2)$ & $27.4(6.9)$ & $37.2(6.3)$ & $23(2)$ \\
\hline Splice & 658 & $80.5(29.5)$ & $98.1(35.6)$ & $89.0(30.4)$ & $95(32)$ & 267 & $11.2(1.9)$ & $13.2(2.5)$ & 13.7 (1.9) & $12(1)$ \\
\hline $\begin{array}{c}\text { Frameshift } \\
\text { indel }\end{array}$ & 1040 & $217.8(112.1)$ & 225.5 (121.7) & $247.2(118.7)$ & 348 (159) & 337 & $38.2(9.2)$ & $36.2(9.0)$ & $44.0(8.0)$ & 38 (11) \\
\hline $\begin{array}{l}\text { Large } \\
\quad \text { deletion }\end{array}$ & 142 & $32.4(12.2)$ & $31.2(11.8)$ & $31.4(9.7)$ & $31(5)$ & 116 & $28.3(6.2)$ & $26.7(5.9)$ & $26.6(5.5)$ & $24(4)$ \\
\hline Total & 2951 & 416.4 (175.6) & $468.2(195.8)$ & $476.7(316.0)$ & $654(286)$ & 1285 & 103.9 & $103.5(24.3)$ & $121.5(21.7)$ & 97 (18) \\
\hline
\end{tabular}


$\left(P<10^{-8}\right.$ for all comparisons) and significantly higher than for missense variants in CEU $(P<$ $0.05)$. Because most variants in a given genome are common, the comparatively high rate of annotation errors among high-frequency LoF variants meant that filtering resulted in a large reduction in LoF variants per individual (Table 1).

We identified several sources of false-positive LoF annotation that will require careful consideration in clinical sequencing projects. For instance, the predicted functional effect of a nonsense or frameshift variant can be altered by other nearby variants on the same chromosome (table S1 and fig. S2), and predicted splice-disrupting SNVs and indels can be rescued by nearby alternative splice sites (fig. S3). Both nonsense SNVs and frameshift indels are enriched toward the $3^{\prime}$ end of the affected gene, consistent with a greater tolerance to truncation close to the end of the coding sequence (Fig. 1C); putative LoF variants identified in the last $5 \%$ of the coding region were thus systematically removed from our highconfidence set, with the single exception of a known LoF indel in the NOD2 gene. There is also a discernible peak close to the $5^{\prime}$ end of genes, suggesting that some disrupted transcripts are rescued by transcriptional reinitiation at an alternative start codon (Fig. 1C).

Notably, 415 (32.3\%) of our high-confidence LoF variants are partial LoF variants, affecting only a subset of the known transcripts from the affected gene, meaning that functional protein may still be produced. We chose not to discard such cases, as it is currently impossible to assess the relative functional importance of different transcripts for most genes, and partial LoF mutations have previously been shown to be causal in Mendelian diseases (11).

In total, $43.5 \%(1285 / 2951)$ of our candidate LoF variants survived filtering. The resulting cat- alog of high-confidence LoF variants is not complete: The 1000 Genomes pilot projects had low power to detect extremely rare variants (2), and we will not have detected certain classes of LoF variants, such as large gene-disrupting duplications, noncoding variants that disrupt gene expression or splicing regulation, or coding variants that destroy protein function without overtly disrupting an open reading frame (such as missense SNVs or in-frame indels). Several known LoF variant-containing genes such as ACTN3 (12) and CASP12 (13) were labeled as "polymorphic pseudogenes," meaning that the reference genome contains nonfunctional allele of the gene, whereas in other haplotypes the gene is functional (14); it is likely that we missed LoF variants in other uncharacterized genes from this class.

Nonetheless, this catalog represents the largest available set of high-confidence human variants predicted to disrupt protein-coding genes. We note that the majority of the LoF variants identified here are novel: $70 \%$ of the high-confidence LoF SNVs and indels were not present in dbSNP before the 1000 Genomes pilot project.

The true number of LoF variants in an individual genome. Using the systematically curated list of variants from NA12878, we estimate that this anonymous individual with European ancestry carries $97 \mathrm{LoF}$ variants, with 18 present in a homozygous state (Table 1 and table S2). These numbers, though still indicating an unexpected tolerance for gene inactivation in humans and being considerably higher than those based on genotyping known nonsense SNVs alone (15), are substantially lower than most previously published estimates based on whole-genome sequencing [e.g., $(2,3,16)$ ] and provide a benchmark for further studies of individual variation in functional gene content. This analysis also provides a robust estimate of different variant classes on gene inactivation: for instance, we find that $39 \%$ of genes inactivated in the NA12878 genome are the result of frame-shifting indels, a potentially serious concern given that indels are typically undercalled using short-read sequencing approaches (2). Over a quarter $(28.7 \%)$ of the LoF SNVs and indels in NA12878 affect only a subset of the known transcripts from the affected genes, emphasizing the need to consider alternative splicing in the annotation of functional effects.

Properties of LoF variants and affected genes. LoF SNVs are markedly enriched for low-frequency alleles compared to synonymous and missense SNVs (Fig. 1A), suggesting that many LoF variants are deleterious to human health and hence are prevented from increasing in frequency by purifying natural selection. The number of highconfidence LoF variants per individual is $25 \%$ higher in the YRI (Nigerian) sample than in the three non-African populations $\left(P=5.0 \times 10^{-21}\right.$; Table 1), suggesting a higher level of variation in functional gene content in African individuals consistent with their greater overall genetic diversity. However, we caution that larger samples with more homogeneous sequencing quality across populations will be required to confirm this finding and assess its likely functional impact.

We compared the properties of genes carrying at least one high-confidence $\mathrm{LoF}$ variant with those of other protein-coding genes. Genes containing high-confidence LoF alleles are relatively less evolutionarily conserved, showing a higher ratio of protein-altering to silent substitutions in coding regions between human and macaque $\left(P=2.8 \times 10^{-52}\right)$ and less evolutionary conservation in their promoter regions (GERP score; $P=$ $\left.3.7 \times 10^{-16}\right)$. On average, they have more closely related gene family members (paralogs) than other genes $(P=0.0058)$ and show greater sequence identity to paralogs $(P=0.0068)$, suggesting that

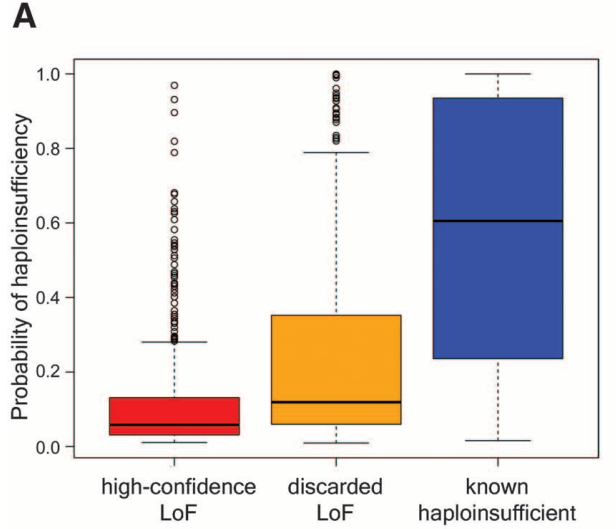

Fig. 2. (A) Estimated probability of haploinsufficiency (presence of disease due to heterozygous loss of function), using a model trained with an independent set of LoF deletions as well as a set of known haploinsufficient genes. (B) Association of coding variants with complex disease risk. Observed $-\log _{10}(P)$ values for disease association in 17,000 individuals from seven complex disease cohorts and a shared control group, following imputation of variants identified by the 1000 Genomes low-coverage pilot, are plotted against the expected null distribution for all LoF variants and
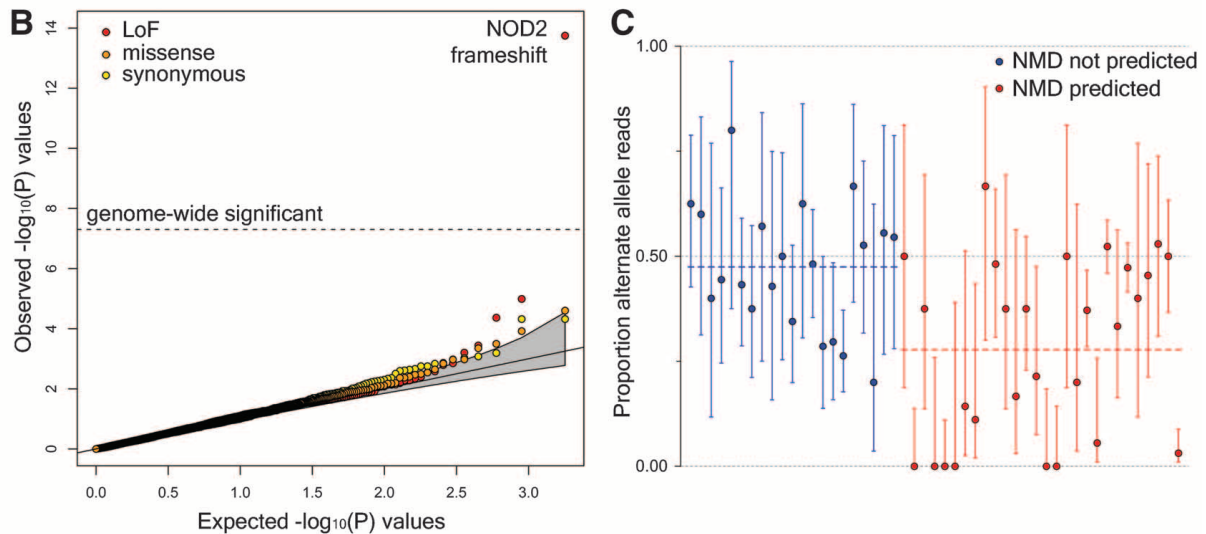

frequency-matched missense and synonymous SNPs. (C) Allele-specific expression analysis of nonsense variants, using RNA sequencing data from 119 lymphocyte cell lines. Circles show the proportion of LoF-carrying reads spanning each site across all heterozygous individuals. Variants predicted to cause nonsense-mediated decay (NMD, red) and those predicted to escape NMD (blue) are arbitrarily ordered by genome position within each class. Blue and red dashed horizontal lines indicate mean values in each class. Error bars, 95\% confidence interval. 
in many cases their function may be partially redundant and also increasing the possibility that LoF variants may be gained or lost through the process of gene conversion (17), as has recently been reported for disease mutations (18). They also have lower connectivity in both proteinprotein interaction $\left(P=6.8 \times 10^{-6}\right)$ and gene interaction $\left(P=4.2 \times 10^{-19}\right)$ networks, suggesting that LoF-containing genes are generally less central to key cellular pathways, although there are caveats to this interpretation (9). LoF-containing genes are strongly enriched for functional categories related to olfactory reception and depleted for genes implicated in protein-binding, transcriptional regulation, and anatomical development (table S8).

We estimated the probability that heterozygous inactivation of a given gene will be deleterious (a state known as haploinsufficiency) using a combination of functional and evolutionary parameters $(9,19)$. Our filtering process disproportionately removed candidate LoF variants with a higher predicted probability of haploinsufficiency, $P(\mathrm{HI})$, consistent with the majority of putative LoF variants in highly functionally constrained genes being artifactual (Fig. 2A). Highconfidence LoF variants remaining after filtering have significantly lower $P(\mathrm{HI})$ than variants discarded by our filters $\left(P=2.1 \times 10^{-16}\right)$ or known haploinsufficient genes $\left(P=1.8 \times 10^{-73}\right)$.

We identified 365 genes with multiple candidate LoF variants. The majority of the genes with three or more independent LoF variants were found to represent systematic sequencing errors: for instance, the $C D C 27$ gene contained 10 separate candidate splice-disrupting variants, all of which were found to represent mapping errors due to an inactive gene copy absent from the human reference sequence. Most of these variants were removed by filtering (table S3). Of the remaining genes, some likely represent genes drifting toward inactivation in the population: for instance, the $V W D E$ gene contains four separate high-confidence LoF variants, with $42.7 \%$ of the sequenced $1000 \mathrm{G}$ samples carrying at least one nonfunctional copy of this gene.

Effects of LoF variants on human phenotypes and disease risk. The high-confidence LoF set includes many known LoF variants reported to have effects on human traits (table S4). We also found a number of previously uncharacterized LoF variants likely to have phenotypic effects. For instance, we identified three separate LoF variants in PKDIL3 and one in PKD2L1; the protein products of these two genes form a putative sour taste receptor complex $(20,21)$, so these variants may underlie variation in sour taste sensitivity between humans.

Our high-confidence LoF set includes many variants relevant to severe human disease. We identified 26 known recessive disease-causing mutations in our high-confidence LoF set, including mutations associated with the severe early-onset conditions Leber congenital amaurosis, harlequin ichthyosis, osteogenesis imperfecta, and Tay-Sachs disease (table S5). We also identified 21 strong candidates for novel disease-causing mutations: high-confidence LoF variants affecting all known transcripts of genes in which other null mutations have been convincingly associated with Mendelian disease, including adult-onset muscular dystrophy, Charcot-Marie-Tooth disease, and mucolipidosis (table S6). With one exception (a variant associated with transplant graft-versus- host disease), no individuals were homozygous for the putative disease-causing alleles.

Given the evidence for the presence of known deleterious variants, we hypothesized that LoF variants may also be enriched for association with risk of common, complex diseases. We investigated this hypothesis by imputing genotypes for $417 \mathrm{LoF}$ SNVs and indels into a total of 13,241 patients representing seven complex diseases such as Crohn's disease and rheumatoid arthritis, along with 2938 shared controls, who had previously been subjected to genome-wide single-nucleotide polymorphism (SNP) genotyping (22). We confirmed a previously known frameshift indel in the NOD2 gene associated with Crohn's disease, with a genome-wide significant imputed $P$ value of $1.78 \times 10^{-14}$ (two orders of magnitude more significant than the best tag SNP). However, no other LoF variants achieved genome-wide significance, and there was no overall excess of association signals in LoF variants compared to other coding variants (Fig. 2B). Because our catalog is expected to contain most genuine $\mathrm{LoF}$ variants at greater than $5 \%$ frequency, this result suggests that common gene-disrupting variants play a minor role in complex disease predisposition.

One explanation for the paucity of common LoF variants associated with complex disease risk is purifying selection, which is expected to prevent most severely deleterious alleles from reaching high population frequencies; this is consistent with the skew toward low frequencies among high-confidence LoF variants (Fig. 1A). In addition, genes containing homozygous LoF variants have more gene family members (median 5 versus $3 ; P=3.76 \times 10^{-3}$ ) and are less

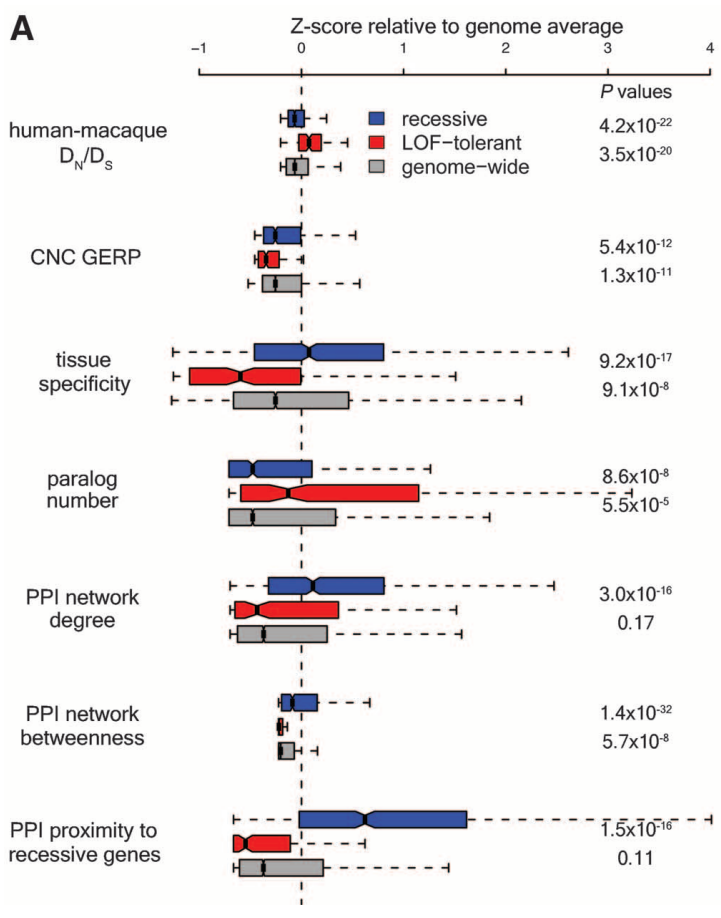

B

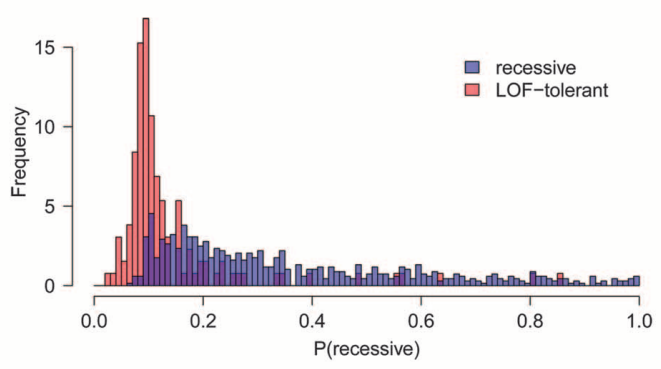

C

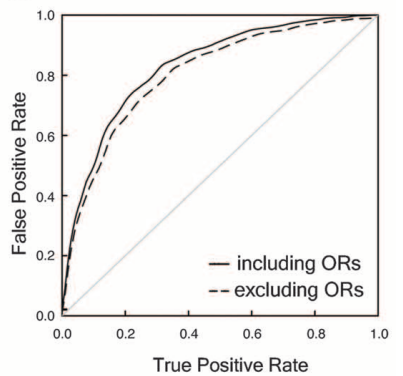

Fig. 3. (A) Distribution of selected evolutionary and functional parameters for recessive disease genes (blue) and LoF-tolerant genes (red) compared to all protein-coding genes (gray). Values are transformed to $z$ scores to allow parameters to be plotted together. Boxes show interquartile range with medians indicated with a vertical black line, and whiskers terminate at the most extreme point less than 1.5 times the interquartile range from the box. For each pair of $P$ values, the top value refers to the recessive versus LoF-tolerant comparison and the bottom value refers to the LoF-tolerant versus genome background comparison. Because many of the parameters are left-skewed, the medians typically fall below zero. (B) $P$ value distribution for linear discriminant model (LDM) trained using LoF-tolerant and recessive disease genes, based on human-macaque $D_{\mathrm{N}} / D_{\mathrm{S}}$ ratio and PPI network proximity to known recessive disease genes. (C) Receiver-operating characteristic (ROC) curve for LDM distinguishing between LoF-tolerant and recessive disease genes, both when olfactory receptor genes (ORs) are included [solid line, area under curve $(A \cup C)=0.831]$ and excluded (dashed line, $A U C=0.814) . D_{N} / D_{S}$, ratio of missense to synonymous substitutions; CNC GERP, GERP score for conserved noncoding elements within $50 \mathrm{~kb}$ of gene; PPI, protein-protein interaction. 
conserved between macaque and human $(P=$ $\left.1.87 \times 10^{-4}\right)$ than genes containing only heterozygous LoF variants, suggesting greater redundancy in genes affected by high-frequency loss of function. Similarly small effects on complex disease risk have previously been noted for large, common copy-number variations, another class of variant with a high prior probability of functional impact (23).

Genotype imputation and case-control association studies have low power to detect associations for low-frequency variants, so further experiments involving direct genotyping of LoF variants in large disease cohorts will be required to characterize the impact of rare LoF variation on human complex disorders.

Effects of nonsense SNVs on gene expression. We examined the impact of validated nonsense SNVs on gene expression using RNA sequencing data generated from lymphoblastoid cell lines of 119 samples from two populations $(24,25)$. Comparison of the relative expression of the LoF and functional alleles within experimentally genotyped heterozygous individuals (Fig. 2C and table S7) revealed a statistically significant reduction in expression from the LoF allele in 8/49 (16.3\%) of variants with sufficient sequencing depth to be assayed. As expected, this reduction in expression is most common for variants predicted to trigger nonsense-mediated mRNA decay (NMD), a cellular process that degrades premature stop codoncontaining transcripts: $7 / 28(25.0 \%)$ of predicted NMD-triggering variants show significant evidence of decay, compared to $1 / 21$ (4.8\%) of predicted NMD-evading variants, and the proportion of reads mapping to the alternate allele was significantly lower for predicted NMD-triggering variants (median 0.352 versus $0.481 ; P=0.0023$ ). However, most predicted NMD-triggering variants have no detectable effect on gene expression.

These results provide functional confirmation of true loss of gene function for a minority of LoF variants. In addition, they demonstrate that the most widely used algorithm for NMD prediction (26) is an imperfect indicator of the effects of nonsense SNVs on RNA expression.

Natural selection on LoF variants. We explored whether LoF variants as a class showed evidence of recent positive selection, as expected under the "less is more" hypothesis of adaptive gene loss proposed by Olson (27). We examined the overlap between high-confidence LoF variants and regions showing potential signatures of positive selection using frequency spectrum and haplotype length-based tests on 1000 Genomes pilot data (2). In contrast to the "less is more" hypothesis, LoF variants overlapped with positively selected regions no more often than frequencymatched synonymous SNVs. However, we have identified 20 high-confidence LoF variants in candidate regions for positive selection that warrant further analysis (table S10).

In some cases, selection for gene inactivation may act through the accumulation of multiple rare LoF variants rather than increased frequency of a specific LoF allele. We identified one potential example of this: In addition to a relatively common nonsense SNV in the CD36 gene reported to be the target of positive selection in African populations (28), we identified two rare, novel splice-disrupting SNVs in the same gene. All three of these variants were specific to the Yoruban (YRI) population, suggesting that multiple null alleles for CD36 may be accumulating in African populations under the influence of selection.

Using LoF-tolerant genes to predict the probability of disease causation for novel variants. Homozygous inactivation of a gene can have a range of phenotypic effects: At one end of the spectrum are severe recessive disease genes, while at the other end are genes that can be inactivated without overt clinical impact, referred to here as LoFtolerant genes. Clinical sequencing projects seeking to identify disease-causing mutations would benefit from improved methods to distinguish where along this spectrum each affected gene lies.

Genes homozygously inactivated in $1000 \mathrm{Ge}-$ nomes Project samples are likely to fall close to the LoF-tolerant end of the spectrum. These genes therefore represent a comparison group that can be used to define the functional and evolutionary characteristics that distinguish these genes from severe recessive disease genes.

We examined the 253 genes containing validated $\mathrm{LoF}$ variants that were found to be homozygous in at least one individual. These LoF-tolerant genes are significantly less conserved and have fewer protein-protein interactions than the genome average (Fig. 3A). They are also enriched for functional categories related to chemosensation, largely explained by the enrichment of olfactory receptor genes in this class $(13.0 \%$ versus $1.4 \%$ genome-wide), and depleted for genes involved in embryonic development and cellular metabolism (table S8).

We then identified parameters that could be used to classify candidate genes along the disease/LoF-tolerant spectrum. We first removed olfactory receptors from the LoF-tolerant set, as these genes could be easily excluded as candidates for most severe Mendelian diseases, leaving $213 \mathrm{LoF}$-tolerant genes to compare with 858 known recessive disease genes. These two gene categories were found to display marked differences in a wide range of properties (Fig. 3A).

We developed a linear discriminant model based on human-macaque conservation and proximity to recessive disease genes in a proteinprotein interaction network to classify genes into LoF-tolerant and recessive disease classes (Fig. 3 , B and C). Although insufficient to definitively discriminate between the two classes, this algorithm could be used to prioritize candidates identified by sequencing recessive disease patients for replication and functional follow-up. We have calculated a recessive disease probability score for each protein-coding gene in the genome for use in such analyses (9).

Conclusions. Here we describe a stringently filtered catalog of variants disrupting the reading frame of human protein-coding genes, including the majority of such variants present at a population frequency of $1 \%$ or greater. Because large numbers of candidate $\mathrm{LoF}$ variants are present in the genomes of all individuals, but are highly enriched for a variety of sequencing and annotation errors, there is a need for caution in assigning disease-causing status to novel gene-disrupting variants found in patients. More reliable reference gene sets will help: Reference sequence and automated gene annotation errors accounted for $44.9 \%$ of candidate LoF variants in our deeply characterized individual genome, but most of these have now been corrected as a result of this project and other manual annotation efforts.

Our stringent filtering of the LoF variants found in a single high-quality human genome suggests that a typical "healthy" genome contains $\sim 100$ genuine LoF variants, with most of them carried in the heterozygous state. Given that humans (29) and other species (30) have been estimated to carry fewer than five recessive lethal alleles per genome, it seems likely that the majority of LoF variants found in an individual genome are common variants in nonessential genes, although these may still have an effect on human phenotypic variation. Nonetheless, the signature of strong purifying selection against high-confidence LoF variants as a class, and the discovery of numerous known and predicted severe recessive disease alleles, indicates that many LoF alleles with large effects on human fitness exist at low frequency in the human population. Large sequencing and genotyping projects will be required to uncover the full spectrum of these variants and their effects on human disease risk.

We have found that LoF-tolerant and recessive disease genes have differing functional and evolutionary properties, allowing us to develop a potential approach for prioritizing novel candidate recessive disease variants identified in patient samples for functional follow-up. As further examples of LoF-tolerant genes are obtained from high-throughput sequencing studies, the power of this type of classification approach is likely to grow considerably.

Finally, we note that our catalog of validated LoF variants comprises a list of naturally occurring "knockout" alleles for more than 1000 human protein-coding genes, many of which currently have little or no functional annotation attached to them. Identification and systematic phenotyping of individuals homozygous for these variants could provide valuable insight into the function of many poorly characterized human genes.

\section{References and Notes}

1. P. C. Ng et al., PLoS Genet. 4, e1000160 (2008).

2. The 1000 Genomes Project Consortium, Nature 467, 1061 (2010).

3. K. Pelak et al., PLoS Genet. 6, e1001111 (2010).

4. D. G. MacArthur, C. Tyler-Smith, Hum. Mol. Genet. 19 (R2), R125 (2010).

5. S. Balasubramanian et al., Genes Dev. 25, 1 (2011).

6. M. A. DePristo et al., Nat. Genet. 43, 491 (2011). 
7. J. Harrow et al., Genome Biol. 7 (suppl. 1), \$4, 1 (2006).

8. http://vat.gersteinlab.org/

9. See supporting material on Science Online.

10. R. E. Mills et al.; 1000 Genomes Project, Nature 470, 59 (2011).

11. A. Uzumcu et al., ]. Med. Genet. 43, e5 (2006).

12. D. G. MacArthur et al., Nat. Genet. 39, 1261 (2007).

13. Y. Xue et al., Am. J. Hum. Genet. 78, 659 (2006).

14. Z. D. Zhang, A. Frankish, T. Hunt, ]. Harrow, M. Gerstein, Genome Biol. 11, R26 (2010).

15. B. Yngvadottir et al., Am. J. Hum. Genet. 84, 224 (2009).

16. J. R. Lupski et al., N. Engl. J. Med. 362, 1181 (2010).

17. J. M. Chen, D. N. Cooper, N. Chuzhanova, C. Férec, G. P. Patrinos, Nat. Rev. Genet. 8, 762 (2007).

18. C. Casola, U. Zekonyte, A. D. Phillips, D. N. Cooper, M. W. Hahn, Genome Res. 10.1101/gr.127738.111 (2011).

19. N. Huang, I. Lee, E. M. Marcotte, M. E. Hurles, PLoS Genet. 6, e1001154 (2010)

20. Y. Ishimaru et al., Proc. Natl. Acad. Sci. U.S.A. 103, 12569 (2006).

21. A. L. Huang et al., Nature 442, 934 (2006).

22. Wellcome Trust Case Control Consortium, Nature 447, 661 (2007).
23. D. F. Conrad et al., Nature 464, 704 (2010). 24. S. B. Montgomery et al., Nature 464, 773 (2010).

25. J. K. Pickrell et al., Nature 464, 768 (2010).

26. E. Nagy, L. E. Maquat, Trends Biochem. Sci. 23, 198 (1998).

27. M. V. Olson, Am. J. Hum. Genet. 64, 18 (1999).

28. A. E. Fry et al., Hum. Mol. Genet. 18, 2683 (2009).

29. A. H. Bittles, J. V. Neel, Nat. Genet. 8, 117 (1994).

30. A. R. McCune et al., Science 296, 2398 (2002).

Acknowledgments: T. Shah provided the Pyvoker software used for manual assignment of genotypes based on intensity clusters; S. Edkins was involved in the Sequenom validation; and the genotyping groups at Illumina, the Wellcome Trust Sanger Institute, and The Broad Institute of Harvard and MIT provided raw intensity data for the three Illumina arrays used for genotyping validation. The work performed at the Wellcome Trust Sanger Institute was supported by Wellcome Trust grant 098051; D.G.M. was supported by a fellowship from the Australian National Health and Medical Research Council; G.L. by the Wellcome Trust (090532/Z/09/Z); E.T.D. and S.B.M. by the Swiss National Science Foundation, the Louis Jeantet Foundation, and the $\mathrm{NIH}-$ National Institute of Mental Health GTEx fund; K.Y. by the Netherlands Organisation for Scientific Research (NWO) VENI grant 639.021.125; and H.Z., Y.L., and J.W. by a National Basic Research Program of China (973 program no. 2011CB809200), the National
Natural Science Foundation of China (30725008, 30890032, 30811130531), the Chinese 863 program (2006AA02A302 2009AA022707), the Shenzhen Municipal Government of China (grants JC200903190767A, JC200903190772A, ZYC200903240076A, CXB200903110066A, ZYC200903240077A and ZY(200903240080A), and the Ole Rømer grant from the Danish Natural Science Research Council, as well as funding from the Shenzhen Municipal Government and the Local Government of Yantian District of Shenzhen. M.B.G. and C.T.-S. contributed equally to this work as senior authors. J.K.P. is on the scientific advisory board of $23 \mathrm{andMe}$, and R.A.G. has a shared investment in Life Technologies. Raw sequence data for the 1000 Genomes pilot projects are available from www. 1000genomes.org, and a curated list of the loss-of-function variants described in this manuscript is provided in the supporting online material.

\section{Supporting Online Material}

www.sciencemag.org/cgi/content/full/335/6070/823/DC1 Materials and Methods

Figs. $\mathrm{S} 1$ to $\mathrm{S} 5$

Tables S1 to S10

References (31-71)

Data Files $S 1$ to $S 3$

10 October 2011; accepted 11 January 2012

10.1126/science. 1215040

\section{Unraveling the Spin Polarization of the $v=5 / 2$ Fractional Quantum Hall State}

\author{
L. Tiemann, ${ }^{1,2 *}$ G. Gamez, ${ }^{1}$ N. Kumada, ${ }^{1}$ K. Muraki ${ }^{1,2 *}$
}

The fractional quantum Hall (FQH) effect at filling factor $v=5 / 2$ has recently come under close scrutiny, as its ground state may possess quasi-particle excitations obeying nonabelian statistics, a property sought for topologically protected quantum operations. However, its microscopic origin remains unknown, and candidate model wave functions include those with undesirable abelian statistics. We report direct measurements of the electron spin polarization of the $v=5 / 2 \mathrm{FQH}$ state using resistively detected nuclear magnetic resonance. We find the system to be fully polarized, which unambiguously rules out the most likely abelian contender and lends strong support for the $v=5 / 2$ state being nonabelian. Our measurements reveal an intrinsically different nature of interaction in the first excited Landau level underlying the physics at $v=5 / 2$.

$\mathrm{W}$

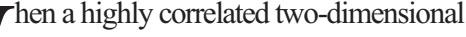
electron system (2DES) is placed under a strong perpendicular magnetic field $B$ at low temperature, the interplay between quantum mechanics and the interelectron interaction produces spectacular effects. When the filling factor $v$, the ratio between the number of electrons $\left(n_{\mathrm{s}}\right)$ and the number of magnetic flux quanta $\left[n_{\phi}=(e / h) B ; e\right.$ : electron charge; $h$ : Planck's constant], takes particular "magic" rational values $p / q$ ( $p, q$ : integer), an energy gap forms based purely on electron correlation and makes the system's transverse (Hall) resistance invariant to small

${ }^{1}$ NTT Basic Research Laboratories, NTT Corporation, 3-1 MorinosatoWakamiya, Atsugi 243-0198, Japan. '2ERATO Nuclear Spin Electronics Project, Japan Science and Technology Agency (JST), Kawaguchi 332-0012, Japan.

*To whom correspondence should be addressed. E-mail: lars.tiemann@gmail.com (L.T.); muraki.koji@lab.ntt.co.jp (K.M.) perturbations (1). In these fractional quantum Hall $(\mathrm{FQH})$ phases, small variations of $n_{\mathrm{s}}$ or $n_{\phi}$ generate quasiparticles with a fractional charge $e^{*}= \pm e / q(2)$. The composite-fermion (CF) model (3), in which an electron is transformed into a fictitious particle by merging it with an even number of flux quanta, explains FQH effects for odd values of $q$. The most notable FQH state with an even denominator observed to date, the $v=5 / 2$ FQH state (4), cannot be described within this simple CF picture, and it has recently come under close scrutiny as it may support something even more tantalizing: a nonabelian state of matter (5-7). Charge excitations from this potential nonabelian state would be carried by quasiparticles whose interchange takes the system from one of its many ground states to another, whereas the interchange of ordinary abelian quasiparticles only adds a phase to their wave functions. This unusual property, specific to nonabelian states, is proposed as the foundation for topological quantum computation that would be robust against environmental decoherence (8). Both nonabelian $(5,9,10)$ and abelian (11) states have been proposed for $v=5 / 2$, but experimental efforts (12-16) have not discriminated clearly between them. Two most probable wave function candidates that emerged through quasiparticle tunneling experiments on $v=5 / 2$ (13) are the nonabelian anti-Pfaffian state $(9,10)$ and the abelian (331) state (11). One can discriminate between them by measuring the spin polarization of the state (17-19), as the former is fully polarized, and the latter unpolarized $(18,19)$.

We perform this measurement using a $100-\mu \mathrm{m}-$ wide Hall bar (Fig. 1A) with the 2DES confined to a 27-nm-wide gallium arsenide (GaAs) quantum well. A back gate enables us to tune the electron density $n$ from $0.5 \times 10^{15}$ to $4.2 \times$ $10^{15} \mathrm{~m}^{-2}$. At $n=4.2 \times 10^{15} \mathrm{~m}^{-2}$, the longitudinal resistance $R_{x x}$ and the Hall resistance $R_{x y}$ show a well-developed $v=5 / 2 \mathrm{FQH}$ state at $12 \mathrm{mK}$, along with pronounced $\mathrm{FQH}$ features at $v=7 / 3$ and $8 / 3$, indicating a high sample quality (20) (Fig. 1B).

Our measurement of the spin polarization exploits the hyperfine interaction that intrinsically exists between the magnetic moments of the atoms constituting the GaAs quantum well and the spins of the electrons confined therein. When the 2DES has a nonzero spin polarization $P$, nuclei in contact with the 2DES experience a local magnetic field, which shifts their nuclear resonance frequency to a lower value by an amount proportional to $P$ (Knight shift $K_{\mathrm{s}}$ ). We measure $K_{\mathrm{s}}$ using the resistively detected nuclear magnetic resonance (RD-NMR) technique (21), where the resonant absorption of radio frequency (rf) and the resultant change in the nuclear polarization 
Research Articles: "A systematic survey of loss-of-function variants in human proteincoding genes" by D. G. MacArthur et al. (17 February, p. 823). In Fig. 3C, the axis labels "False Positive Rate" and "True Positive Rate" should have been swapped. The corrected figure is shown here. The figure has been corrected in the HTML version online.

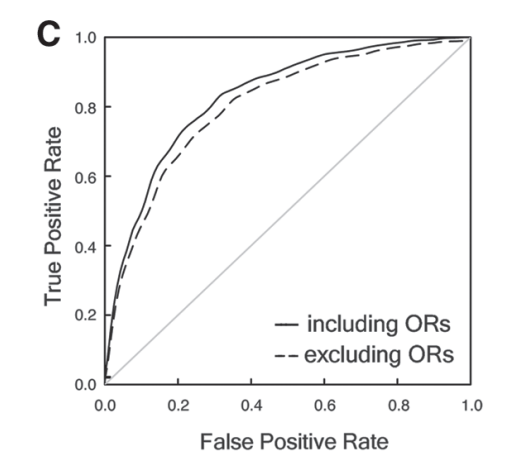


Daniel G. MacArthur, Suganthi Balasubramanian, Adam Frankish, Ni Huang, James Morris, Klaudia Walter, Luke Jostins, Lukas Habegger, Joseph K. Pickrell, Stephen B. Montgomery, Cornelis A. Albers, Zhengdong D. Zhang, Donald F. Conrad, Gerton Lunter, Hancheng Zheng, Qasim Ayub, Mark A. DePristo, Eric Banks, Min Hu, Robert E. Handsaker, Jeffrey A. Rosenfeld, Menachem Fromer, Mike Jin, Xinmeng Jasmine Mu, Ekta Khurana, Kai Ye, Mike Kay, Gary Ian Saunders, Marie-Marthe Suner, Toby Hunt, If H. A. Barnes, Clara Amid, Denise R. Carvalho-Silva, Alexandra H. Bignell, Catherine Snow, Bryndis Yngvadottir, Suzannah Bumpstead, David N. Cooper, Yali Xue, Irene Gallego Romero, 1000 Genomes Project Consortium, Jun Wang, Yingrui Li, Richard A. Gibbs, Steven A. McCarroll, Emmanouil T. Dermitzakis, Jonathan K. Pritchard, Jeffrey C. Barrett, Jennifer Harrow, Matthew E. Hurles, Mark B. Gerstein and Chris Tyler-Smith (February 16, 2012)

Science 335 (6070), 823-828. [doi: 10.1126/science.1215040]

Editor's Summary

\section{Defective Gene Detective}

Identifying genes that give rise to diseases is one of the major goals of sequencing human genomes. However, putative loss-of-function genes, which are often some of the first identified targets of genome and exome sequencing, have often turned out to be sequencing errors rather than true genetic variants. In order to identify the true scope of loss-of-function genes within the human genome,

MacArthur et al. (p. 823; see the Perspective by Quintana-Murci) extensively validated the genomes from the 1000 Genomes Project, as well as an additional European individual, and found that the average person has about 100 true loss-of-function alleles of which approximately 20 have two copies within an individual. Because many known disease-causing genes were identified in "normal" individuals, the process of clinical sequencing needs to reassess how to identify likely causative alleles.

This copy is for your personal, non-commercial use only.

Article Tools

Permissions
Visit the online version of this article to access the personalization and article tools:

http://science.sciencemag.org/content/335/6070/823

Obtain information about reproducing this article: http://www.sciencemag.org/about/permissions.dtl

Science (print ISSN 0036-8075; online ISSN 1095-9203) is published weekly, except the last week in December, by the American Association for the Advancement of Science, 1200 New York Avenue NW, Washington, DC 20005. Copyright 2016 by the American Association for the Advancement of Science; all rights reserved. The title Science is a registered trademark of AAAS. 